\title{
Auf dem Weg zu einem ökologischen Gesellschaftsvertrag in feministischer Tradition
}

\author{
Arbeit beansprucht natürliche Ressourcen und \\ prägt soziale Verhältnisse. Um den Weg zu einer \\ geschlechtergerechten und ökologischen \\ Gesellschaft zu ebnen, ist das Konzept der Arbeit \\ ausschlaggebend. Welche Veränderungen wären \\ nötig, um eine solche gesellschaftliche Trans- \\ formation zu bewirken? \\ Von Clara Wolff, Santje Kludas und Nina Prehm
}

Lohnarbeitszeit pro Person erreicht werden soll. Gleichzeitig werden Maßnahmen wie ein bedingungsloses Grundeinkommen (BGE) gefordert, die das damit sinkende Lohneinkommen kompensieren sollen - zumindest für Gering- und Mittelverdienende (attac 2018). Zudem sollten die bei einzelnen Arbeiter/innen wegfallenden Arbeitsstunden in Neueinstellungen anstelle einer Intensivierung der geleisteten Arbeit münden (Flecker et al. 2014). Mit einer Lohnarbeitszeitverkürzung (LZV) und einem daran gekoppelten BGE könne man, so das Argument, somit sowohl eine geringere Erwerbslosenquote auch bei insgesamt sinkendem Bedarf an Arbeitsstunden - als auch eine gerechtere Verteilung von Wohlstand, Zeit und Arbeit und damit eine höhere Lebensqualität für alle erreichen. Wie kann dieser Vorschlag aus einer feministischen Perspektive beurteilt werden?

\section{Lohnarbeitszeitverkürzung als nachhaltige Alternative zum Wachstumsimperativ?}

Um beurteilen zu können, inwiefern die LZV tatsächlich eine Alternative zum Wachstumsimperativ darstellen kann, muss zuerst die ökologische Bedeutung von Arbeit an sich charakterisiert werden. Diese liegt zum einen in ihrer Rolle als vermittelnde Instanz zwischen Mensch und Natur im Rahmen der Produktion (Biesecker 2000). Durch Arbeit eignet sich der Mensch die Natur an, um auf ihrer Basis Güter und Dienstleistungen zu produzieren, die seine Bedürfnisse befriedigen. Bei gleichbleibender Produktivität gilt, je mehr gearbeitet wird, desto mehr wird auch produziert. Im Umkehrschluss heißt das, dass eine wachsende Produktion einen durch Effizienzsteigerungen sinkenden Bedarf an Arbeitskräften kompensiert. Stetiges Wirtschaftswachstum wird als vermeintlich einzige Lösung erachtet, um Erwerbslosigkeit entgegenzuwirken. Solange jedoch keine absolute Entkopplung von Ressourcenverbrauch und Bruttoinlandsprodukt gelingt, bedeutet Wachstum eine steigende Umweltbelastung. So werden soziale und ökologische Fragen häufig gegeneinander ausgespielt. Im vorherrschenden politischen Diskurs werden dabei selten Fragen nach einer Umverteilung von Einkommen und Arbeit gestellt. Eine solche Umverteilung könnte auch im Falle einer gleichbleibenden Produktion Erwerbslosigkeit vorbeugen. Somit entkräftet eine LZV mit Einkommensausgleich die scheinbare Alternativlosigkeit ökologisch fragwürdiger Wachstumsstrategien als Mittel gegen Erwerbslosigkeit. 
Zum anderen kann eine LZV Lebensstile verändern. Mehr Zeit könnte zu suffizienteren Lebensweisen, beispielweise durch mehr Selbstversorgung sowie lokalen Konsum und damit zu einer nachhaltigen Entwicklung beitragen (Schor 2016). Erste Untersuchungen weisen jedoch darauf hin, dass mehr Zeit mitunter auch deutlich ressourcenintensiveren Konsum befördert - gerade bei Haushalten mit geringerem Einkommen (Buhl 2015; Schor 2016). Diese Wirkungen werden auch unter Zeit- und Einkommens-Rebound-Effekten zusammengefasst. Um dies zu verhindern, sollten Finanzierungsmodelle des Einkommensausgleichs in Betracht gezogen werden, die auf Abgaben auf umweltschädlichen Aktivitäten basieren.

Auch aus feministischer Sicht gibt es viele Gründe, eine LZV zu befürworten. Unter den richtigen Bedingungen ermöglicht sie mehr Menschen den Zugang zum Erwerbsarbeitsmarkt, ohne dabei im Gegenzug „das Ganze der Arbeit“ (Biesecker 2000) - also auch die Sorge um andere, die Persönlichkeitsentwicklung, freiwilliges Engagement sowie politische Teilhabe vernachlässigen zu müssen. Dies trägt zu mehr Sichtbarkeit und Anerkennung dieser Formen der Arbeit bei. Zudem stellt eine LZV die Basis einer gerechteren Verteilung von Reproduktionsarbeit dar und hinterfragt traditionelle, geschlechterspezifische Rollenverteilungen. Ob eine Arbeitszeitverkürzung tatsächlich sozial und ökologisch nachhaltiger ist, hängt vor allem von der Form des Lohnausgleichs für niedrige und mittlere Einkommen ab. Ein Lohnausgleich kann entweder durch einen erhöhten Mindestlohn erreicht werden, der dazu führt, dass man mit verringertem Stundensatz trotzdem genug verdienen kann. Alternativ könnte eine Entkopplung von Arbeit und Einkommen mittels eines bedingungslosen Grundeinkommens (BGE) erfolgen.

\section{Einkommensausgleich durch ein bedingungsloses Grundeinkommen}

Aus feministischer Perspektive wird die Einführung eines BGE zwiespältig gesehen (Robeyns 2001). Auch wenn die tatsächlichen gesellschaftlichen Auswirkungen eines BGE schwer zu prognostizieren sind, ist anzunehmen, dass Menschen, die unbezahlte Arbeit leisten, kurzfristig finanziell bessergestellt würden. Davon würden unter derzeitigen Verhältnissen insbesondere Frauen profitieren, die aufgrund des sogenannten „Gender Care Gap“ entweder unter einer enormen Doppelbelastung durch Lohn- und Sorgearbeit leiden oder nur geringfügig erwerbstätig sind. Zugleich stoßen Männer weiterhin auf große Widerstände, wenn sie sich um eine Verringerung der Lohnarbeitszeit bemühen, um ihre Kinder oder Eltern zu pflegen (Gräff 2018). Gerade Frauen bleiben dadurch häufig in finanzieller Abhängigkeit von Familienangehörigen oder dem Sozialsystem, die durch ein individuell ausgezahltes BGE reduziert würde. Das Problem fehlender gesellschaftlicher Wertschätzung von Sorgearbeit bliebe jedoch auch mit einem BGE bestehen, da es nicht spezifisch an diese Tätigkeiten gekoppelt ist.
Inwiefern ein BGE zu einer Minderung des bestehenden Gender Care Gaps führt, ist umstritten. Das BGE ermöglicht es Menschen, freier zwischen bezahlten und unbezahlten Tätigkeiten zu wählen, was generell als emanzipatorisch angesehen werden kann. Insbesondere könnte ein Anreiz entstehen, dass mehr Männer ihre Erwerbsarbeitszeit zugunsten von Reproduktionsarbeit reduzieren. Es wäre jedoch auch möglich, dass sich unter derzeitigen gesellschaftlichen Verhältnissen überwiegend Frauen aus dem Erwerbsarbeitsmarkt zurückziehen würden, was geschlechterspezifische Arbeitsaufteilung verstärkt. Das mag zwar den Präferenzen einzelner Frauen entsprechen, kann aber auch zu einer statistischen Diskriminierung von Frauen mit Präferenz für Erwerbsarbeit führen. Dies jedoch als feministisches Gegenargument zu einem BGE anzuführen, ist fragwürdig, da die geschlechterspezifische Arbeitsverteilung nicht ursächlich im BGE, sondern in gesellschaftlichen und historischen Rahmenbedingungen liegt. Deshalb auf ein BGE zu verzichten, würde im Umkehrschluss bedeuten, Frauen aus monetären Gründen in die Erwerbsarbeit zu drängen, was insbesondere bei prekären Jobs nicht besonders emanzipatorisch sein kann. Ein BGE kann also durchaus emanzipatorische Wirkungen entfalten - solange es mit Maß nahmen zur Befreiung von Menschen aus den beschriebenen Rollenmustern einhergeht.

Folglich müssen eine LZV und ein BGE in einen neuen Gesellschaftsvertrag eingebettet werden, der das Modell des männlichen Brotverdieners auflöst. Fraser argumentiert, dass dieses Modell zwar an Bedeutung verliere, es dennoch bis heute die Wohlfahrtsstaaten Europas und Nordamerikas dominiere (Fraser 1994). Trotz sich verändernder institutioneller Rahmenbedingungen wie Steuersysteme, Rechte auf Elternzeit oder Teilzeitarbeit, seien keine grundlegenden Veränderungen in der Geschlechtergerechtigkeit zu beobachten. Als konzeptionelles Gegengewicht entwirft Fraser daher das Universal Care Model. Es entstammt der feministischen Wohlfahrtsstaatsforschung, die davon ausgeht, dass ein geschlechtergerechter Wohlfahrtsstaat nicht nur bezahlte Arbeit anerkennen muss, sondern unbezahlte Arbeit im Haushalt und Betreuungstätigkeiten (Care) in den Mittelpunkt aller Strategien rücken sollte. Somit berücksichtigt das Modell das Grundbedürfnis aller Menschen, in ihrem Leben sowohl umsorgt zu werden als auch für andere $\mathrm{zu}$ sorgen - das Recht auf und zu Sorgearbeit.

\section{Ein gendergerechter und ökologisch nachhaltiger Gesellschaftsvertrag ist möglich}

Aufbauend auf der feministischen Kritik Frasers hat das Netzwerk „Vorsorgendes Wirtschaften“ bereits seit Anfang der 1990er Bedingungen erforscht, unter denen ein neuer gendergerechter und zugleich ökologisch nachhaltiger Gesellschaftsvertrag möglich wäre. Das Konzept beinhaltet neben einem erweiterten Arbeitsbegriff sowie der Umverteilung von Arbeit und Einkommen auch die „Neuordnung von Geschlechterund Generationenverhältnissen“ (Biesecker 2000). Zugleich 
stellt das Konzept drei Prinzipien ins Zentrum allen Handelns: 1) Orientierung am Lebensnotwendigen statt an monetären Größen 2) Kooperation statt Konkurrenz und 3) Vorsorge statt Nachsorge (Jochimsen et al. 1994). In Orientierung an diese Prinzipien folgt das Konzept der Idee, dass viele „kleine neue Gesellschaftsverträge“ (Biesecker 2000) schrittweise eine größere sozial-ökologische Transformation herbeiführen. Erste Beispiele für solche kleinen neuen Gesellschaftsverträge sind kooperative Formen des Wirtschaftens in Repair Cafés, Kollektiven oder Gemeinschaftsgärten. Um diese Initiativen zu stärken und dabei auch der ökologischen Nachhaltigkeit gerecht zu werden, müssen politische Maßnahmen getroffen werden: Die hier diskutierten Ansätze der LZV in Kombination mit Einkommensausgleich durch ein BGE und einem Wohlfahrtsstaat, der Sorgearbeit als gleichwertig anerkennt, wären ein Anfang. Begleitet durch Anreize für nachhaltige Produktionsweisen und Konsummuster, einen Wandel der Lebensstile und eine Bildung, die die Bürger/innen befähigt, kooperativ und vorsorgend $\mathrm{zu}$ agieren, könnten sie Grundlage eines ökologischen Gesellschaftsvertrags in feministischer Tradition sein, der sowohl die Befreiung aus traditionellen Rollenmustern als auch das Einhalten der planetaren Grenzen beinhaltet.

\section{Literatur}

attac (2018): ArbeitFair Teilen. www.attac-netzwerk.de/ag-arbeitfairteilen/ startseite

Biesecker, A. (2000): Kooperative Vielfalt und das Ganze der Arbeit: Überlegungen zu einem erweiterten Arbeitsbegriff. Berlin, Wissenschaftszentrum Berlin für Sozialforschung.

Bundesministerium für Familie, Senioren, Frauen und Jugend (BMFSFJ) (2017): Zweiter Gleichstellungsbericht der Bundesregierung.

Buhl, J. (2015): Steigerung und Beschleunigung: Zeit-Rebound-Effekte nach Arbeitszeitverkürzung. In: Ökologisches Wirtschaften 30/4: 28-29.

Federici, S. (2004): Caliban And The Witch: Women, The Body, and Primitive Accumulation. New York, Autonomedia.
Flecker, J./Altreiter, C. (2014): Warum eine Arbeitszeitverkürzung sinnvoll ist. Linz, Institut für Sozial- und Wirtschaftswissenschaften.

Fraser, N. (1994): After the Family Wage. Gender Equity and the Welfare State. In: Political Theory 22 (4): 591-618.

Gräff, F. (2018): Einfach ein bisschen weniger. In: taz (20.4. 2018). www.taz. de/!5497729

Jochimsen, M. (1994). Vorsorgendes Wirtschaften. Konturenskizze zu Inhalt und Methode einer ökologischen und sozialverträglichen Ökonomie. In: Radloff, J./Busch-Lüty, C./Knobloch, U./Seidl, I. (Hrsg.): Vorsorgendes Wirtschaften: Frauen auf dem Weg zu einer Ökonomie der Nachhaltigkeit. Politische Ökologie (Sonderheft 6). München, oekom.

Robeyns, I. (2001): Will a Basic Income Do Justice to Women? In: Analyse \& Kritik 23 (1): 88-105.

Schor, J. (2016): Wahrer Wohlstand. Mit weniger Arbeit besser Leben. München, oekom.

Statistisches Bundesamt (2014): 69\% der berufstätigen Mütter arbeiten in Teilzeit. www.destatis.de/DE/PresseService/Presse/Pressemitteilungen/ zdw/2014/PD14_013_p002pdf.pdf?_blob=publicationFile

Steffen, W. et al. (2015) „Planetary Boundaries: Guiding Human Development on a Changing Planet“. In: Science 347 (6223).

\section{AUTORINNEN + KONTAKT}

Clara Wolff, Santje Kludas und Nina Prehm sind Mitarbeiterinnen am Institut für ökologische Wirtschaftsforschung (IÖW) und interessieren sich seit ihrem Studium für feministische Ökonomiekritik und Nachhaltigkeit.

Institut für ökologische Wirtschaftsforschung, $\mathrm{GmbH}$ (gemeinnützig), Potsdamer Str. 105, 10785 Berlin.

Tel.: +4930 8845940. E-Mail: santje.kludas@ioew.de, nina.prehm@ioew.de, clara.wolff@ioew.de, Website: www.ioew.de/service/impressum
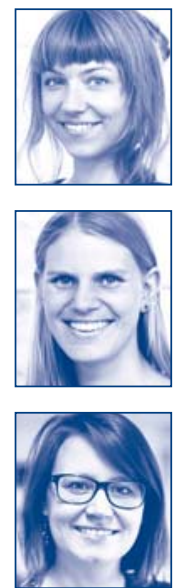

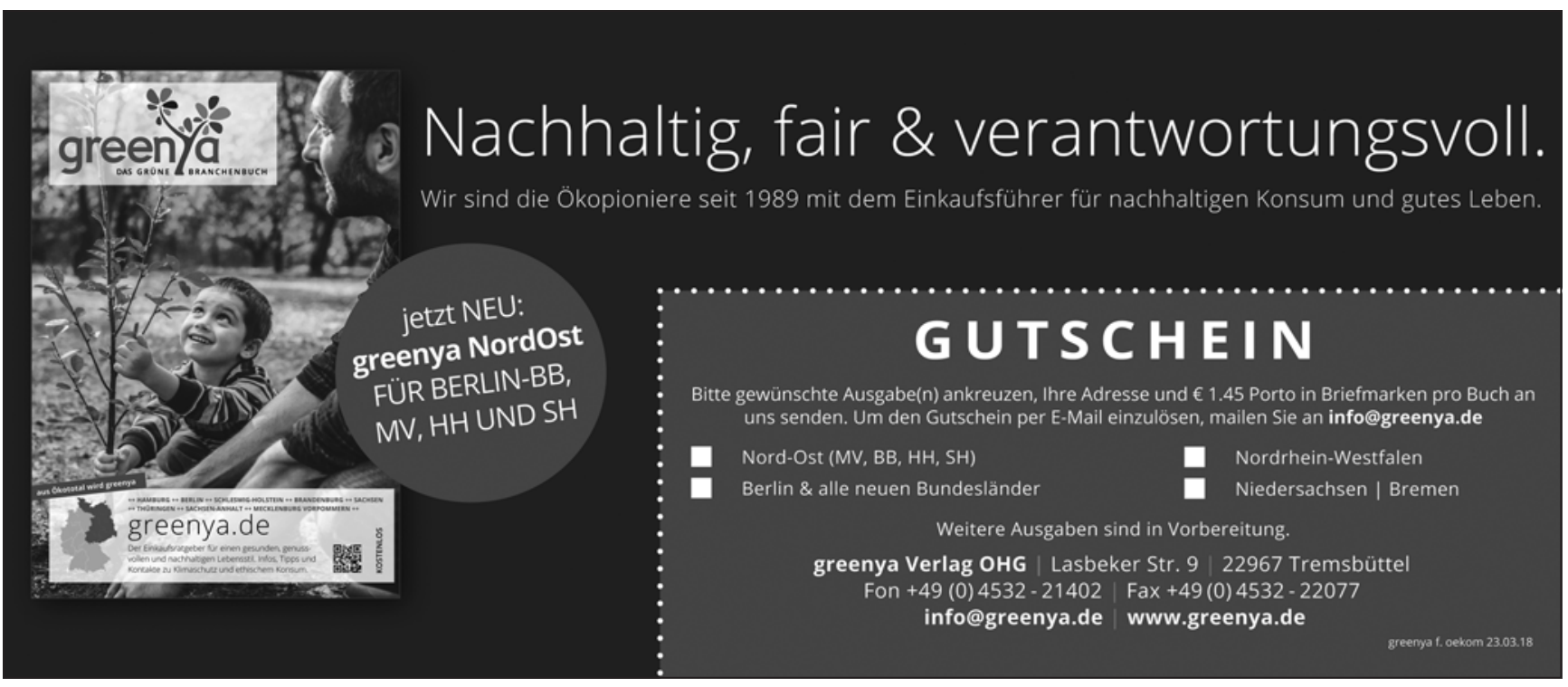

